\title{
The first study of genetically regulated potato starch biochemical characteristics for $S$. tuberosum cultivars of russian selection
}

\author{
V. Khlestkin ${ }^{1,2}$, T. Erst ${ }^{1}$, L. Gvozdeva ${ }^{1}$, Yu. Khoroshavin ${ }^{1,2}$, I. Totsky ${ }^{1}$, \\ E. Khlestkina ${ }^{1,2}$ \\ ${ }^{1}$ Institute of Cytology and Genetics SB RAS, Novosibirsk, Russia \\ ${ }^{2}$ Novosibirsk State University, Novosibirsk, Russia \\ *e-mail:khlestkin@bionet.nsc.ru
}

Key words: potato starch, morphology, amylose, amylopectin, resistant starch, phosphorylation

\begin{abstract}
Motivation and Aim: Starch is a well-known sustainable plant resource, which is important for food, textile, paper and other industries. Starch consists of two polysaccharides linear amylose and branched amylopectin, packed in semicrystalline granules in plant plastids. Ease of isolation makes it an economical and readily available source of pure carbohydrates. Exact ratio and structure of the polysaccharides significantly influence the macroscopic practically useful physical and chemical properties of starch gels. Potato is the most reasonable source of starch in Siberia. Thus, knowledge about gene networks regulating potato starch biosynthesis is a key to growth and manufacturing starch with target properties.
\end{abstract}

Methods and Algorithms: 90 Cultivars of potato (Solanum tuberosum L.) mostly of Russian selection from ecological-geographical testing and "GenAgro" collection grown in 2017 in Novosibirsk region were treated to isolate DNA and starch. Wide SNPgenotyping of the DNA isolated has been outsourced for further association studies. Some important biochemical parameters of starch were evaluated. Thus, we developed a procedure for microscopy analysis of starch granules morphology. We also compiled and tuned a procedure for evaluation of amylose and amylopectin content in potato starch by spectrophotometry. "Resistant starch" value for a raw potato starch for various cultivars and phosphorylation of starch polysaccharides were also estimated.

Results: For the first time an advanced biochemical analysis of a wide range of potato cultivars of Russian selection has been performed. Morphological traits, amylose amylopectin ratio, resistant starch, glucose units phosphorylation were evaluated. We were able to identify contrasting forms on every biochemical parameter studied for the given set of $S$. tuberosum cultivars. These data will be used for both DNA markers elaboration for marker - assisted selection and future genome-wide association studies. Some assumptions on DNA loci, responsible for starch biochemical traits in the studied cultivars, may be done based on the literature data.

Conclusion: We shown that amylose content of the studied set of $S$. tuberosum cultivars varies within 13-30\%, phosphorus content - within $0.05-0.1 \%$, resistant starch within 40-99\%. Average starch granule value is in significant correlation with starch preparative yield. Moderate correlations of resistant starch content with Feret's diameter and phosphorylation with preparative starch yield are observed.

Acknowledgements: Supported by RFBR (17-29-08006). Potato cultivars obtained from plant collection "GenAgro" (ICG SB RAS). 